Modern Physics Letters A

(C) World Scientific Publishing Company

\title{
DUALISM BETWEEN PHYSICAL FRAMES AND TIME IN QUANTUM GRAVITY
}

\author{
SIMONE MERCURI \\ ICRA International Center for Relativistic Astrophysics c/o Dipartimento di Fisica (G9) \\ Università di Roma "La Sapienza", Piazza A.Moro 500185 Rome, Italy. \\ mercuri@icra.it \\ GIOVANNI MONTANI \\ ICRA - International Center for Relativistic Astrophysics c/o Dipartimento di Fisica (G9) \\ Università di Roma "La Sapienza", Piazza A.Moro 500185 Rome, Italy. \\ Dipartimento di Fisica Università di Roma "La Sapienza". \\ montani@icra.it \\ Received (Day Month Year) \\ Revised (Day Month Year)
}

\begin{abstract}
In this work we present a discussion of the existing links between the procedures of endowing the quantum gravity with a real time and of including in the theory a physical reference frame.

More precisely, as first step, we develop the canonical quantum dynamics, starting from the Einstein equations in presence of a dust fluid and arrive to a Schrödinger evolution. Then, by fixing the lapse function in the path-integral of gravity, we get a Schrödinger quantum dynamics, of which eigenvalues problem provides the appearance of a dust fluid in the classical limit.

The main issue of our analysis is to claim that a theory, in which the time displacement invariance, on a quantum level, is broken, is indistinguishable from a theory for which this symmetry holds, but a real reference fluid is included.
\end{abstract}

Keywords: Quantum gravity; reference frame.

PACS Nos.: 04.60.-m

\section{General Statements}

Gravitational interaction in view of its non linear nature has to find its most appropriate quantum approach in the path-integral representation, in fact there are many formal analogies between gravitational and non Abelian gauge theories, especially when the former is represented in a connection formalism 11. Furthermore in General Relativity there is no chance to speak of a well defined perturbation theory, since free asymptotic states in strict sense do not exist, in fact unlike Yang Mills fields, the gravitational one is the geometry of the space-time and should couple locally with any physical system. So that the notion of "in" and "out" states loses 
its physical meaning. However it is worth noting that a satisfactory description of the quantum field theory on a fixed background including the graviton-matter interaction is provided by the modern approach of string theory 1817; nevertheless to extend the quantization procedure to the whole geometry of the space-time is a problem which, up to now, did not find a solution within the superstring framework. Promising formalisms toward the non perturbative quantization of the space-time structure appeared in the first nineteenth, when the loop quantum gravity theory, 22021 , was settled down. Indeed the spin networks formalism provides well defined states for the canonical quantum gravity theory and can be upgraded to a path-integral procedure by virtue of the recent spin foam approach 317 .

The first attempt for a path-integral approach to quantum gravity was due to Hawking et al. $\frac{698}{9}$ in the early eighties. They addressed this problem from a rather heuristic point of view, in fact their path-integral had not a definite Lebesgue measure and even its integrand was ill defined.

Our point of view is that one of the main problems in both the canonical and path-integral approaches to quantum gravity is the non existence of evolution for the quantum dynamics. We think that this problem is strictly connected with the choice of a physical reference system.

The aim of this work is, in fact, to show how there exists a dualism, in quantum gravity, between introducing a real reference fluid and breaking down the invariance of the theory under time displacements. The contact issue in these two points of view consists in providing a physical time for the quantum dynamics, 121910541141316, (for applications see 222315).

To achieve this result, we first show, in "section 2", that to quantize the gravitational field in presence of a dust reference fluid implies to define an appropriate time variable as the conjugate momentum to the eigenvalue of the super-Hamiltonian. Indeed such a non-zero eigenvalue is due to the dust contribution and, being in general non positive, allows us to claim that: a reference fluid, in quantum gravity, is never a test one and its energy density can be even negative.

Then, in "section 3", the picture is completed by fixing the lapse function in the path-integral for the gravitational field, so outlining that it leads, in first approximation, to a Schrödinger dynamics. Via the eigenvalues problem, we finally arrive, in the classical limit, to show the appearance of a dust fluid.

In section 4 brief concluding remarks follow.

\section{Gravity-Fluid Quantization}

In treating General Relativity, we commonly refer to a reference frame as to a specific system of coordinates. However, these two notions are significantly different, because it is necessary, to speak of a real reference frame, the introduction in the space of a material framework (at least locally), to which physically link the description of the phenomena. In General Relativity a real reference frame is implemented considering a fluid which fills the whole space-time (or isolated regions) by its world lines; of 
course more general physical entities can be used to define a reference frame, but they can be reduced to the primitive notion of a fluid.

To specify a reference fluid we have to assign a 4 -velocity vector field describing the world lines of each fluid element; being a physical system, the fluid has always associated a non zero energy-momentum tensor. Indeed, it is just in neglecting the energy-momentum carried by the fluid the main source of confusion between the notions of reference frame and of coordinates system. The latter can be regarded as simply labeling the points of $\mathcal{M}^{4}$ and it is well implemented by a "test fluid". Let us now emphasize a crucial difference existing between the classical and quantum dynamical role played by a real reference fluid. Below we will discuss the case of a dust, which plays the role of reference fluid. In view of its phenomenological nature, the canonical quantization procedure is developed by inferring the Hamiltonian constraints from the Einstein equations.

Let us consider a 4-dimensional space-time manifold, $\mathcal{M}^{4}$, on which a coordinates system $\left\{y^{\mu}\right\}$ and a metric tensor $g_{\mu \nu}\left(y^{\rho}\right)$ (with the signature -+++ , where $\mu, \nu, \rho=$ $0,1,2,3)$ are assigned. A dust fluid is characterized by its proper energy density $\varepsilon\left(y^{\rho}\right)$ and the 4-velocity $u^{\mu}\left(y^{\rho}\right)\left(g_{\mu \nu} u^{\mu} u^{\nu}=-1\right)$, which leads to the energy-momentum tensor $T_{\mu \nu}=\varepsilon u_{\mu} u_{\nu}$.

The Einstein equations and the conservation laws, for the coupled gravity-fluid system take the form:

$$
\begin{aligned}
& G_{\mu \nu}=\chi \varepsilon u_{\mu} u_{\nu}, \\
& u^{\nu} \nabla_{\nu} u^{\mu}=0, \\
& \nabla_{\nu}\left(\varepsilon u^{\nu}\right)=0,
\end{aligned}
$$

where $G_{\mu \nu}$ and $\chi$ denote respectively the Einstein tensor and constant.

We can decompose the metric tensor as follows:

$$
g_{\mu \nu}=h_{\mu \nu}-u_{\mu} u_{\nu} \Rightarrow h_{\mu \nu} u^{\nu}=0 .
$$

Remembering a well-known result, it is easy to show that the following relations take place 2421

$$
\begin{aligned}
& G_{\mu \nu} u^{\mu} u^{\nu}=-\frac{H\left(h_{i j}, p^{i j}\right)}{2 \sqrt{h}}=\chi \varepsilon, \\
& G_{\mu \nu} u^{\mu} h_{i}^{\nu}=\frac{H_{i}\left(h_{i j}, p^{i j}\right)}{2 \sqrt{h}}=0 .
\end{aligned}
$$

Here $h_{i j}(i j=1,2,3)$ denotes the 3-metric of the spatial hypersurfaces orthogonal to $u^{\mu}$ (requiring the condition $u_{[\mu} \nabla_{\nu} u_{\rho]}=0$ the Frobenius theorem (see ${ }^{24}$ p.434) assures the existence of the hypersurfaces orthogonal to $\left.u_{\mu}\right), h \equiv \operatorname{deth}_{i j}$ and $p^{i j}$ its conjugate momentum, while $H$ and $H_{i}$ refer respectively to the super-Hamiltonian and the super-momentum for the gravitational field. Indeed, because of its phenomenological nature we do not deal with the Lagrangean formulation for the fluid and, therefore, the above relations hold if we make the reasonable assumption that the conjugate momentum $p^{i j}$ be not affected by the matter variables (i.e. the fluid 
term in ADM formalism should not contain the time derivative of the 3-metric tensor). These equations remain valid in any system of coordinates, being the dependence on the lapse function and the shift vector contained in the remaining Einstein equations. However only the Hamiltonian constraints are relevant for the quantization procedure and, in the comoving frame, when the 4-velocity becomes $u^{\mu}=\{1, \mathbf{0}\}\left(N=1 N^{i}=0\right)$, we have to retain also the conservation law

$$
\varepsilon \sqrt{h}=-\frac{\omega\left(x^{i}\right)}{2 \chi}
$$

where $x^{i}$ denotes the spatial coordinates of the comoving frame and $\omega\left(x^{i}\right)$ a generic 3 -scalar density of weight $1 / 2$. Here, a crucial point relies on the synchronous nature of the comoving frame as a consequence of the geodesic motion of the dust fluid (12) reduces to an identity, because of the comoving form of $u^{\mu}$ ).

Thus, when the coordinates system becomes a real physical frame, the Hamiltonian constraints read

$$
H=\omega\left(x^{i}\right), \quad H_{i}=0 .
$$

Now, to assign a Cauchy problem for such a system, in which equations (8) play the role of constraints on the initial data, corresponds to provide on a (non-singular) space-like hypersurface, say $\Sigma^{(0)}$, the values $\left\{h_{i j}^{(0)}, p^{(0) i j}, \varepsilon^{(0)}\right\} ; \omega^{(0)}$ can be calculated, by (8), from these values .

It follows that, by specifying a suitable initial condition, the value of $\omega^{(0)}$ can be made arbitrarily small; from the constraints point of view, a very small value of $\omega^{(0)}$ means, where $h^{(0)}$ is not so, that the fluid becomes a test one (being $\omega$ a constant of the motion). We emphasize that for finite values of $\omega, h$ should not vanish to avoid unphysical diverging energy density of the fluid.

The canonical quantization of this system is achieved as soon as we implement the canonical variables into quantum operators and annihilate the state functional $\Psi$ via the Hamiltonian operator constraints. Thus the quantum dynamics obeys the following eigenvalue problem:

$$
\widehat{H} \Psi\left(\left\{h_{i j}\right\}, \omega\right)=\omega \Psi\left(\left\{h_{i j}\right\}, \omega\right),
$$

where $\left\{h_{i j}\right\}$ refers to a whole class of 3 -geometries, so that the super-momentum constraint automatically holds.

In the above equation (9), the spatial function $\omega$ plays the role of the superHamiltonian eigenvalue; in this respect, we observe how its values can no longer be assigned by the initial conditions, but they have to be determined via the spectrum of $\widehat{H}$. We conclude that, in the quantum regime, a real dust reference fluid never approaches a test system and, in view of the super-Hamiltonian structure (the supermetric has no definite sign), its energy density is not always positive.

In order to understand the physical meaning of including a real dust fluid in quan- 
tum gravity, let us take the Fourier transform of $\Psi$ as

$$
\chi\left(\left\{h_{i j}\right\}, \tau\right)=\int D \omega \Psi\left(\left\{h_{i j}\right\}, \omega\right) \exp \left\{-\frac{i}{\hbar}(\omega \tau)\right\},
$$

denoting by $D \omega$ the measure and by $\tau$ a continous space function.

In view of equation (10), the functional $\chi$ must satisfy the Schrödinger-like equation

$$
i \hbar \partial_{\tau} \chi\left(\left\{h_{i j}\right\}, \tau\right)=\widehat{H} \chi\left(\left\{h_{i j}\right\}, \tau\right) .
$$

The Schrödinger-like character of the above equation, allows us to infer that the quantum dynamics of the gravitational field acquires a time evolution, as soon as, it is constrained to physical frames.

\section{Path-Integral Approach to Hyper Time}

In this section we introduce a real time in the quantum theory by defining a pathintegral in which the lapse function is fixed; then we show how the eigenvalues problem associated with the Schrödinger evolution provides, in the semi classical limit, the energy density of a dust reference fluid. To this end, we adopt for the gravitational field the following path-integral:

$\left|h_{i j}^{(2)}, \Sigma_{(2)}^{3}\right\rangle=\int D h^{(1)} D p^{(1)} \int_{\mathcal{F}} D \Omega \exp \frac{i}{\hbar}\left\{\left(S+S^{\star}\right)+2 \int_{\partial M^{4}} d^{3} x \sqrt{h} k\right\}\left|h_{i j}^{(1)}, \Sigma_{(1)}^{3}\right\rangle$,

calculated on all the possible path which connect the 3 -metric $h_{i j}^{(1)}$ on the hypersurface $\Sigma_{(1)}^{3}$ to the 3 -metric $h_{i j}^{(2)}$ on the hypersurface $\Sigma_{(2)}^{3}$, moreover an integration over all the possible initial configuration for the 3 -metric is performed. In (12) $S$ is the ADM action of the gravitational field and we have added the new term:

$$
S^{\star}=\frac{1}{16 \pi} \int_{t_{0}}^{t} d t^{\prime} \int_{\Sigma_{t^{\prime}}^{3}} d^{3} x \sqrt{h} \Lambda\left(N-N^{*}\right)
$$

which allows us to fix the Lapse function to the fixed value $N^{*}$, through a Lagrange multipliers $\Lambda$; in view of its structure such a term satisfies the appropriate constraints algebra:

$$
\begin{gathered}
\left\{H_{i}(x), H_{k}(y)\right\}=H_{k}(x) \delta_{, i}(x-y)-(i x \rightarrow k y), \\
\left\{H_{k}(x), \tilde{H}(y)\right\}=\tilde{H}(x) \delta_{, k}(x-y), \\
\{\tilde{H}(x), \tilde{H}(y)\}=H^{k}(x) \delta_{, k}(x-y)-(x \rightarrow y),
\end{gathered}
$$


where $\tilde{H}=H-\sqrt{h} \Lambda$. Finally we include a boundary term, $S_{b}$, because we assume non zero normal derivatives on the compact the 3-hypersurfaces $\Sigma_{t}^{3}$.

Our path-integral describes a quantum theory in which the time displacements invariance is explicitly broken, the reason for this approach relies on its equivalence with including a reference fluid in the dynamics. The meaning of the coordinates $\left(t, x^{i}\right)$ will turn out at the end of our procedure, being related to the fluid labels.

The functional integral is taken over the domain $\mathcal{F}$, which is the product of the functional spaces corresponding to the 3 -metric with non vanishing determinant $(h \neq 0)$, to the conjugate momentum, lapse function, shift vector and $\Lambda$ 's; therefore for the Lebesgue measure we have $D \Omega \equiv D \Lambda D p^{k l} D h_{i j} D N^{a} D N$ (in what follows, if not complete, the Lebesgue measure will appear with lower labels of the variables to which it refers).

As soon as we evaluate the functional integral over $\Lambda$, we arrive to the new path-integral:

$$
\begin{aligned}
& \left|h_{i j}^{(2)}, \Sigma_{(2)}^{3}\right\rangle=\int D h^{(1)} D p^{(1)} \int_{\mathcal{F}} D \Omega \exp \frac{i}{\hbar}\left\{S+S^{\star}+S_{b}\right\}\left|h_{i j}^{(1)}, \Sigma_{(1)}^{3}\right\rangle= \\
& =\int D h^{(1)} D p^{(1)} \int_{\mathcal{F}_{p h N N^{i}}} D \Omega_{p h N N^{i}} \delta\left[\sqrt{h}\left(N-N^{*}\right)\right] \exp \frac{i}{\hbar}\left\{S+S_{b}\right\}\left|h_{i j}^{(1)}, \Sigma_{(1)}^{3}\right\rangle= \\
& =\int D h^{(1)} D p^{(1)} \int_{\mathcal{F}_{p h N^{i}}} D \Omega_{p h N^{i}}\left(\prod_{t, x^{k}} \frac{1}{\sqrt{h}}\right) \exp \frac{i}{\hbar}\left\{S\left(N^{*}\right)+S_{b}\right\}\left|h_{i j}^{(1)}, \Sigma_{(1)}^{3}\right\rangle,
\end{aligned}
$$

where $\prod_{t, x^{i}}(h)^{-1 / 2}$, in the last line, comes out from the evaluation of the delta function and $S_{b}$ denotes the boundary term.

Since now the wave functional will depend on $N^{*}$ explicitly, then it changes passing from a 3-hypersurface to a neighboring one, so acquiring a "time" evolution. We will indicate the parameter of this evolution with the label " $t$ ". Therefore taking the two hypersurfaces corresponding to $t$ and $t+\varepsilon(\varepsilon \ll 1)$, which are two different instants of "time" referred to two neighboring hypersurfaces, i.e. to two values of the function $N^{*}$. The expansion of the left and right side of the equation (17) leads to:

$$
\begin{aligned}
& \left|h_{i j}^{t+\varepsilon}, \Sigma_{t+\varepsilon}^{3}\right\rangle=\left|h_{i j}^{t}, \Sigma_{t}^{3}\right\rangle+\varepsilon \partial_{t}\left|h_{i j}^{t}, \Sigma_{t}^{3}\right\rangle= \\
& =\frac{1}{A} \int D h^{(1)} D p^{(1)} \int_{\mathcal{F}_{p}} D \Omega_{p N^{i}}\left(1-\frac{i}{\hbar} \varepsilon \mathcal{H}\right) \exp \left\{\frac{i}{\hbar} \int d^{3} x p^{i j}\left(h_{i j}^{t+\varepsilon}-h_{i j}^{t}\right)\right\}\left|h_{i j}^{t}, \Sigma_{t}^{3}\right\rangle,
\end{aligned}
$$

where: $\frac{1}{A}=\int_{\mathcal{F}_{h}} D h \prod_{t, x^{i}}(h)^{-1 / 2} \exp \left\{-i S_{b} / \hbar\right\}$ (for neighboring hypersurfaces $\Sigma_{t}^{3}$ and 
$\Sigma_{t+\varepsilon}^{3}$ the functional integral over $\mathcal{F}_{h}$ reduces to a fixed value) and we approximate the time derivative of the variable $h_{i j}$ as $\partial_{t} h_{i j} \longrightarrow\left(h_{i j}^{t+\varepsilon}-h_{i j}^{t}\right) / \varepsilon$. Now, replacing the momentum terms by the corresponding functional derivatives, i.e.

$$
\begin{array}{r}
-i \hbar \frac{\delta}{\delta h_{i j}^{t}} \int D p \exp \left\{\frac{i}{\hbar} \int d^{3} x p^{i j}\left(h_{i j}^{t+\varepsilon}-h_{i j}^{t}\right)\right\}= \\
=\int D p p^{i j} \exp \left\{\frac{i}{\hbar} \int d^{3} x p^{i j}\left(h_{i j}^{t+\varepsilon}-h_{i j}^{t}\right)\right\},
\end{array}
$$

the leading term of the expansion above leads to the Schrödinger-like equation:

$$
i \hbar \partial_{t}\left|h_{i j}^{t}, \Sigma_{t}^{3}\right\rangle=\widehat{\mathcal{H}}\left|h_{i j}^{t}, \Sigma_{t}^{3}\right\rangle
$$

where $\widehat{\mathcal{H}}=\int_{\Sigma_{t}^{3}} d^{3} x\left(N^{*} \widehat{H}+N^{k} \widehat{H}_{k}\right)$. In the above formula (20) as well as in (11) the

Hermitian character of $\widehat{H}$ requires the same normal ordering introduced in 1413 . Furthermore in analogy to the proof presented in $\underline{8}$, we can get $\widehat{H}_{k}\left|h_{i j}^{t}, \Sigma_{t}^{3}\right\rangle=0$. Now defining the wave functional $\Psi\left(t,\left\{h_{i j}\right\}\right.$ ) (where with the notation $\left\{h_{i j}\right\}$ we indicate the 3-geometries) as the projection of the ket $\left|h_{i j}^{t}, \Sigma_{t}^{3}\right\rangle$ on the functional space, it is easy to realize that if we expand $\Psi\left(t,\left\{h_{i j}\right\}\right)$ as follows:

$$
\Psi\left(t,\left\{h_{i j}\right\}\right)=\int_{y_{t}^{*}} D \omega \Theta(\omega) \xi_{\omega}\left(\left\{h_{i j}\right\}\right) \exp \left(-\frac{i}{\hbar} \int_{t_{0}}^{t} d t^{\prime} \int_{\Sigma_{t}^{3}} d^{3} x(N \omega)\right),
$$

then the following eigenvalues problem takes place:

$$
\widehat{H} \xi_{\omega}\left(\left\{h_{i j}\right\}\right)=\omega\left(x^{j}\right) \xi_{\omega}\left(\left\{h_{i j}\right\}\right) .
$$

Above we regarded $\xi_{\omega}$ as taken on the 3 -geometries in order to consider the constraint $\widehat{H}_{k} \xi_{\omega}=0$. Now, toward the classical limit, we choose the wave functional in the form: $\psi \propto \exp \{i \sigma\} / \hbar$; then interpreting the $\delta \sigma / \delta h_{i j}$ as the conjugate momentum $p^{i j}$ (this is allowed by the Hamilton-Jacobi structure of the leading order term in $\hbar$ ), we easily recognized that the above eigenvalues problem reduces to the classical Hamiltonian constraints:

$$
H=\omega, \quad H_{k}=0 .
$$

Since the following relations hold

$$
\begin{gathered}
G_{\mu \nu} u^{\mu} u^{\nu}=-\frac{H}{2 \sqrt{h}}=-\frac{\omega}{2 \sqrt{h}}, \\
G_{\mu \nu} u^{\mu} h_{i}^{\nu}=\frac{H_{i}}{2 \sqrt{h}}=0,
\end{gathered}
$$

(where $u^{\mu}$ is the orthonormal vector to the 3 -hypersurfaces), then in the comoving reference $\left(u^{\mu}=(1,0)\right.$, i.e. $\left.N=1, N^{i}=0\right)$ we may write the $0-0$ and $0-j$ Einstein equation:

$$
G_{00}=\chi \varepsilon, \quad G_{0 j}=0,
$$


with $\varepsilon=-\omega / 2 \chi \sqrt{h}$ and by (44) $h_{i}^{\mu}=\delta_{i}^{\mu}$. Finally the variation of $S^{*}$ with respect to $h_{i j}$ does not contribute any term to the Einstein equation, because the variation with respect to $\Lambda$ requires $N=N^{*}$; therefore we may also write $G_{i j}=0$. By the covariance principle of Einstein theory, it follows that in a generic coordinates system, we should re obtain the equations (11). We adopted exactly the same letters $\left(u^{\mu}, \varepsilon, \omega\right)$ to denote the quantity of previous and present section, just to stress the complete equivalence of the two opposite paths: from fluid to Schrödinger equation, from Schrödinger equation to fluid. We conclude this section by stessing that the meaning of the coordinates $\left(t, x^{i}\right)$ in the Schrödinger equation (20) is provided by the fluid interpretation. In fact for $N=1, N^{i}=0$, such coordinates coincide respectively with the "clock" and the elements of the fluid, while in general they are reparameterization of such physical coordionates.

\section{Concluding Remarks}

The most puzzling feature of the Wheeler-DeWitt equation consists in its frozen formalism, i.e. of the absence of any evolution along the family of hypersurfaces $\Sigma_{t}^{3}$, which fulfills the whole space-time. Such a feature is a direct consequence of implementing the time displacements invariance of the theory and prevents a consistent interpretation of the resulting quantum dynamics. Here we have shown how if we break down this invariance on a quantum level, then, in the classical limit, we approach an Einstein dynamics in presence of a dust reference fluid. Our proof is based on the equivalence between a Schrödinger-like dynamics and the Einstein dust equations; this feature suggests that, in quantum gravity, the phenomenology has to be linked to a physical frame. To say better, in quantum regime makes no longer sense to speak simply of coordinates system, because any fluid can not be a test one.

A subtle consequence of such a statement is the appearance of a non positive energy density in the theory. Though it calls attention for deeper understanding, we claim that this feature is a natural implication of the super-Hamiltonian structure and should not be rejected as non physical one.

Finally, we observe that the equivalence of the approaches presented in sections 2 and 3 could not seem complete, because in the former we have in (11) a local Schrödinger dynamics, involving a physical time $\tau$, while in the latter the dynamics is smeared and the label time $t$ appears (20). But, by smearing equation (11) times $N^{*}$ and using the super-momentum constraint, we find:

$$
i \hbar \int_{\Sigma_{t}^{3}} d^{3} x \frac{\delta \chi}{\delta \tau} N^{*}=\widehat{\chi} .
$$

Hence observing that, in view of the construction of section $2, \tau$ denotes a synchronous time, it follows the relation $N^{*}=\partial_{t} \tau$. Therefore, the complete equivalence of the two schemes, relies on the natural identification $\partial_{t}(\ldots) \equiv \int_{\Sigma_{t}^{3}} d^{3} x \frac{\delta(\ldots)}{\delta \tau} \partial_{t} \tau$. We 
conclude by stressing how the smeared dynamics got in this work, exactly coincides with that one presented in 14 , which was based on the presence of the Kinematical term 111413 in the gravity action.

The convergence of these two different approaches support the validity of their physical implications. A final point calls attention for a deeper understanding: The theory developed in section 3 spontaneously breaks the time displacements invariance, while to include the refence fluid as in section 2 preserves the 4-diffeomorphisms; how can these two approaches be equivalent?

The answer to this question relies on the statement of section 2, that a reference fluid in quantum gravity can never be a test one; thus, on a quantumn level, only systems of coordinates associated with fluids, which are source for the space-time curvature, are admissible. If we deal with a single fluid coupled to the 3 -metric then it becomes a "privileged" one, because all other systems of coordinates are simple reparameterization of the physical coordinates linked to this fluid. On a classical level the covariance is restated because any other system of coordinates is a physical frame, when thought as a test fluid. In this sense to consider a reference fluid in quantum gravity is equivalent to break down the time diffeomorphisms and provide a time evolution for the dynamics.

1. Ashtekar, Phys. Rev. Lett. 57, 2244-2247, (1986).

2. A.Ashtekar, C.Rovelli, L.Smolin, Phys. Rev. D44, 1740-1745, (1991).

3. J.W.Barrett, L.Crane, J. Math. Phys. 39, 3296-3302, (1997).

4. J.Bicák, K.Kuchař Phys. Rev. D56, 4878-4895, (1997).

5. J.D.Brown, K.Kuchař Phys. Rev. D51, 5600-5629, (1995).

6. G.W.Gibbons, S.W.Hawking, Phys. Rev. D15, 2752, (1977).

7. M.B.Green, J.Schwarz, E.Witten, Superstring Theory, Cambridge University Press, (1987).

8. J.B.Hartle, S.W.Hawking, Phys. Rev. D28, 2960, (1983).

9. S.W.Hawking, Phys. Rev. D18, 1747, (1978).

10. C.J.Isham, available gr-qc/9201011, (1992).

11. K. Kuchař, Canonical methods of quantisation, (1981), C. Isham, R. Penrose, D. Sciama, eds, 'Quantum Gravity 2: A Second Oxford Symposium', Clarendon Press, Oxford, pp.329-374.

12. K.Kuchař, C.Torre, Phys. Rev. D43, 419-444, (1991).

13. S.Mercuri, G.Montani, to appear on Int. Journ. Mod. Phys. D, (2003).

14. G.Montani, Nucl. Phys. B634, 370, (2002).

15. G.Montani, Int. Journ. Mod. Phys. D12, 1445, (2003).

16. H.Nikolić available gr-qc/0106091, (2003).

17. D.Oriti, to appear in Rep. Prog. Phys., available gr-qc/0106091, (2001).

18. J.Polchinski, String Theory, vol.1: Introduction to the Bosonic String, vol.2: Superstring Theory and Beyond, Cambridge University Press, (1998).

19. C.Rovelli, Class. and Quantum. Grav. 8, 297 and 317, (1991).

20. C.Rovelli, available gr-qc/9710008, (1997).

21. T.Thiemann, available gr-qc/0110034, (2001).

22. C.Vaz, L.Witten, Phys. Rev. D60, 024009, (1999).

23. C.Vaz, L.Witten, T.P.Singh, Phys. Rev. D63, 104020, (2001).

24. R.M.Wald, General Relativity, The University of Chicago Press, (1984). 


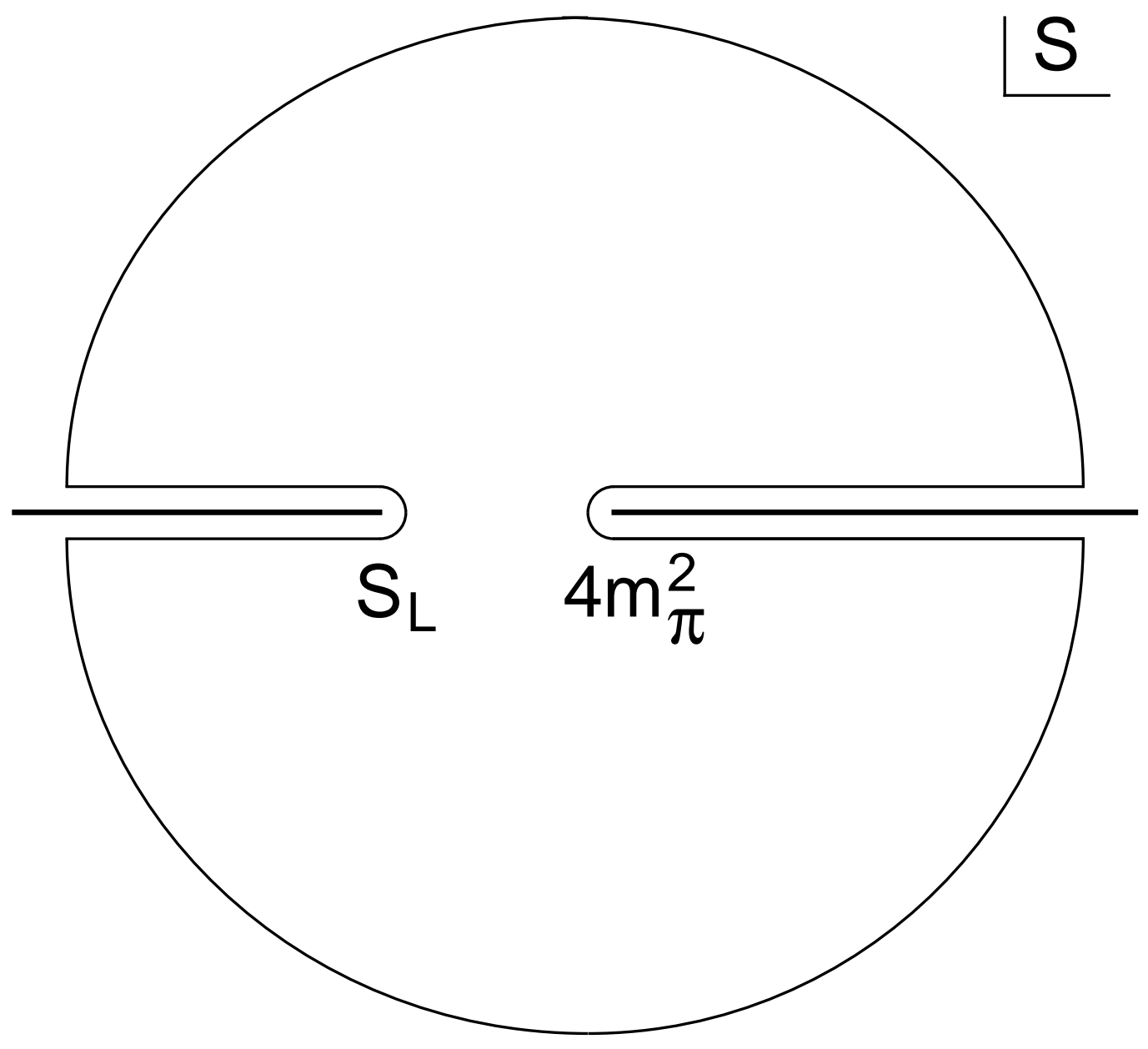

